le portiQue Le Portique

le portiQue Revue de philosophie et de sciences humaines

$11 \mid 2003$

Le Respect

\title{
Autorité et respect en éducation
}

\section{Eirick Prairat}

\section{OpenEdition}

\section{Journals}

Édition électronique

URL : http://journals.openedition.org/leportique/562

DOI : 10.4000/leportique.562

ISSN : $1777-5280$

\section{Éditeur}

Association "Les Amis du Portique"

Édition imprimée

Date de publication : 1 janvier 2003

ISSN : 1283-8594

\section{Référence électronique}

Eirick Prairat, «Autorité et respect en éducation », Le Portique [En ligne], 11 | 2003, mis en ligne le 15 décembre 2005, consulté le 25 mars 2021. URL : http://journals.openedition.org/leportique/562 ; DOI : https://doi.org/10.4000/leportique.562

Ce document a été généré automatiquement le 25 mars 2021

Tous droits réservés 


\title{
Autorité et respect en éducation
}

\author{
Eirick Prairat
}

1 Je voudrais montrer, dans ce court article, qu'il y a une dialectique silencieuse au cœur du travail éducatif et que cette dialectique, qui est celle de l'autorité et du respect, prend une résonance toute particulière dans le contexte actuel de crise de l'éducation.

2 Le débat sur l'autorité s'est cristallisé, ces vingt dernières années, sur des positions diamétralement opposées. D'un côté, ceux qui stigmatisent l'autorité comme une figure douce de la violence. L'autorité est, pour eux, synonyme d'empire, d'emprise, de domination. Celui qui exerce l'autorité est toujours un dominus, c'est-à-dire un maître au sens de celui qui entend maîtriser. De l'autre côté, il y a ceux qui pensent que l'autorité libère. La supprimer reviendrait purement et simplement à supprimer la possibilité même d'éduquer. C'est aussi notre avis. Sans doute, faut-il déjà s'entendre sur les mots.

\section{La Potestas et l'Auctoritas}

3 La distinction latine entre potestas et auctoritas permet une première précision. La potestas est le pouvoir fondé sur la fonction, le grade ou le statut. C'est le pouvoir légal, reconnu et accordé par les instances supérieures de la société (militaires, judiciaires, scolaires...). La potestas est le pouvoir de prendre des décisions, de commander, d'exiger l'obéissance dans un domaine donné en recourant à la contrainte, le cas échéant. Le professeur est investi d'une potestas, c'est-à-dire d'un pouvoir légalement reconnu pour exercer sa fonction, il a notamment le droit, droit institutionnellement défini et encadré, de réprimander un élève si le besoin s'en fait sentir.

L'auctoritas ne dépend d'aucune instance. On n'investit pas quelqu'un d'une auctoritas. Elle émane de la personne, c'est ce que confirme l'étymologie puisque le terme est dérivé d'auctor, l'auteur, celui qui est cause première. L'auteur d'une chose est celui qui est à l'origine de ladite chose. L'auctoritas, c'est l'influence, l'ascendant, le crédit. Elle n'est pas fondée sur la puissance légale de contraindre mais sur le prestige de la personne. Elle est l'art d'obtenir l'obéissance sans recours à la menace ou à la contrainte. Elle produit les effets de la force tout en étant le contraire même de la force. Notons que ces deux formes d'autorité - la potestas et l'auctoritas - ne s'impliquent pas nécessairement. On peut avoir de l'influence sans avoir un statut ou une fonction 
légalement reconnue. À l'inverse on peut être investi d'une autorité légale et être dépourvu de toute forme d'auctoritas. Cela étant, elles ne s'excluent pas nécessairement non plus, on peut imaginer qu' " avoir l'autorité » et "avoir de l'autorité » peuvent en certaines circonstances, et fort heureusement, se conjuguer. Si nous entendons maintenant autorité au sens d'auctoritas, nous pouvons alors dire sans jouer sur les mots que l'autoritaire est précisément la personne qui manque d'autorité ou encore, et ce serait plus juste, qu'elle est la personne qui abuse de la potestas pour combler son déficit d'auctoritas.

6 Si l'autorité (au sens d'auctoritas) permet d'obtenir le consentement de son vis-à-vis sans le recours à la force, est-ce à dire qu'elle l'obtient par les moyens de la raison? Si l'autorité ne contraint pas, peut-on dire qu'elle persuade? «L'autorité, écrit Hannah Arendt, exclut l'usage de moyens extérieurs de coercition; là où la force est employée, l'autorité proprement dite a échoué. L'autorité, d'autre part, est incompatible avec la persuasion qui présuppose l'égalité et opère par un processus d'argumentation. Là où on a recours à des arguments l'autorité est laissée de côté. Face à l'ordre égalitaire de la persuasion se tient l'ordre autoritaire, qui est toujours hiérarchique. S'il faut vraiment définir l'autorité, alors ce doit être en l'opposant à la fois à la contrainte par force et à la persuasion par arguments... » ${ }^{1}$. L'autorité n'est ni contrainte, ni persuasion, elle est influence. Dès lors, une nouvelle question se pose : qu'est-ce qui distingue l'influence éducative (l'autorité) de l'influence manipulatrice?

Les caractéristiques de l'autorité éducative

$7 \quad$ L'influence éducative (ou autorité) a quatre grandes caractéristiques.

8 - C'est une influence libératrice. Autorité vient du mot auctor qui lui-même est dérivé du verbe augere (augmenter). L'autorité fait croître, elle augmente l'enfant, d'un mot elle fait grandir. C'est une influence positive. En ce sens, on peut dire d'elle qu'elle est un service.

9 - C'est une action indirecte. L'autorité éducative ne s'épuise pas dans sa transitivité. Elle n'est pas une action sur mais une activité qui vise à susciter, en l'autre, une activité. Elle n'est pas une volonté qui s'oppose et s'impose à une autre volonté pour la soumettre, mais une volonté qui s'allie à une volonté naissante pour l'aider à vouloir. Elle est moins là pour régenter une volonté que pour éclairer une liberté qui se cherche.

10 - C'est une influence temporaire. Cette troisième caractéristique découle des deux précédentes. Alors que l'influence manipulatrice ne cesse de réaffirmer l'asymétrie originelle pour se perpétuer, l'influence éducative travaille à sa propre éclipse. C'est précisément parce qu'elle entend faire advenir un alter ego qu'elle est circonscrite dans le temps.

11 - Enfin, elle présuppose la reconnaissance. L'autorité n'a pas son fondement dans un acte de soumission aveugle mais, tout au contraire, dans un acte de reconnaissance. "L'autorité, écrit Hans-Georg Gadamer, repose sur la reconnaissance [...], sur un acte de raison même qui, consciente de ses limites, accorde à d'autres une plus grande perspicacité ${ }^{2}$. Telle est la faiblesse et la grandeur de l'autorité : être une influence qui pour se déployer comme influence requiert d'être reconnue par celui-là même qui en sera le bénéficiaire.

12 Mais de quelle Autorité, l'autorité éducative se réclame t-elle? En d'autres termes, qu'est-ce qui autorise l'autorité à se donner comme telle? On ne peut éluder cette 
ultime question, celle de « la fondation d'avant la fondation », selon la formule de Paul Ricœur ${ }^{3}$.

De l'Autorité du monde

13 L'éducation appelle l'autorité, avons-nous dit en commençant, il faut en fait retourner cette proposition et dire : c'est parce qu'il y a de l'Autorité qu'il y a de l'éducation. Le monde est toujours déjà là, la culture nous précède et nous enveloppe de toutes parts. Il y a une précédence du monde ; il y a une antériorité, une antécédence de la culture qui fait de l'éducateur un passeur. L'éducateur permet à celui qui vient au monde d'être de ce monde, il accueille et introduit le nouveau venu ou encore, pour reprendre la belle expression de Locke, il «ouvre doucement la scène du monde " ${ }^{4}$. S'il faut insister sur un mot, c'est sur l'adverbe doucement qu'il faut mettre l'accent.

14 L'autorité rend le monde plus accessible en le rendant plus léger, la culture plus proche en la rendant plus lisible. "L'enseignant, en sa pauvre autorité, écrit Marcel Gauchet, n'est jamais qu'un représentant de cette autorité sans visage... Il est en position de médiateur vis-à-vis d'elle... Il vous protège en ce quelle peut avoir d'écrasant, il la rend familière, accessible, argumentable, discutable. Il fonctionne comme un avocat des nouveaux venus auprès de cette source intraitable; il leur ménage des accès et des transactions particulières avec elle ${ }^{5}$.

15 Sans autorité, le monde écraserait le nouveau venu ; sans autorité, la culture, loin d'être une source signifiante pour penser son rapport à soi, aux autres et au monde, deviendrait bruit et non-sens. Ce que les détracteurs modernes de l'autorité oublient c'est cette vérité anthropologique : on n'entre jamais seul dans le monde. À sans cesse rabattre la relation d'autorité sur sa dimension psychologique, à la critiquer à partir des seules théories de l'influence et de la manipulation, ils en oublient une donnée éducative majeure : on ne s'autorise jamais seul à être contemporain du monde.

La figure de l'autorité éducative

Quelle est donc la figure concrète de l'autorité éducative? L'autorité de l'éducateur n'est pas celle de l'arbitre qui tire son crédit de sa posture d'indépendance. L'éducateur, contrairement à l'arbitre, est intéressé par le résultat. Son autorité n'est pas celle du leader car il y a dans celle-ci quelque chose qui est de l'ordre de l'emprise dans cette autorité soucieuse de se maintenir. Elle ressemble parfois à celle de l'expert. Nous savons que l'expert tient son prestige de sa compétence technique ou de son savoir, mais à la différence de l'autorité de l'expert, celle du professeur ou de l'éducateur est une invitation au partage, une "autorisation " à entrer et à être de ce monde. Aussi, l'autorité éducative prend-elle la forme d'une autorité de référence.

Elle est ce à quoi le nouveau venu se réfère pour pouvoir se situer et s'orienter ; ce n'est pas l'autorité du modèle qui appelle la copie et l'imitation et qui finalement engage à devenir autre, comme l'Autre, mais celle d'une instance qui, en dernier lieu, le renvoie toujours à lui-même. Il en est ainsi de ce que l'on appelle un ouvrage de référence, il est un ouvrage dans lequel nous puisons des idées, des intuitions et des orientations mais qui ne nous fera jamais faire l'économie d'une pensée personnelle. La référence est la nécessaire sortie hors de soi, le détour qui nous renvoie à nous-même.

Cette première description met l'accent sur le mouvement d'émancipation, minorant l'autre facette, celle de l'arrachement ; car après tout on entre toujours dans un monde en en quittant un autre. Aussi une seconde manière de donner corps à l'idée d'autorité éducative est de dire qu'elle est une autorité d'adulte. Mais qu'est-ce qu'être adulte ? Ce qu'il importe ici ce n'est pas de définir l'adulte d'un point de vue positif mais de 
comprendre ce que signifie être adulte du point de vue de la valeur. L'adulte, en tant que valeur, ne s'oppose ni à l'enfance ni à l'adolescence, mais à l'infantile qui est un ensemble de traits qui amarre l'enfance au monde de la puérilité ${ }^{6}$.

- L'infantile refuse de voir les choses telles qu'elles sont et refuse de distinguer ce qu'il sait et ce qu'il croit. Il se plaît à prendre ses désirs pour des réalités, tel est son rapport au monde.

20 - L'infantile est incapable de s'abstraire du présent et par conséquent ne veut jamais les moyens des fins qu'il se donne. En somme, il veut sans vouloir, il est velléitaire car il se laisse engluer dans l'immédiateté, tel est son rapport au temps.

21 - L'infantile est égocentrique, fermé à la rencontre car celle-ci comporte toujours une part d'aléa et de risque, tel son rapport frileux à l'altérité.

22 - L'infantile ne sait pas choisir, il ne veut pas choisir car choisir c'est renoncer (à ce que l'on n'a pas choisi) puis assumer (ce que l'on a choisi). L'infantile est indécis, irrésolu, tel est son rapport à lui-même.

23 - L'infantile appréhende le pouvoir soit sur le mode de la révolte, soit sur le mode de la sacralisation. Il oscille entre révolte aveugle et soumission fanatique, tel est son rapport immature au pouvoir.

24 L'adulte comme valeur, comme figure libératrice, s'oppose à ces formes d'attachement et d'aliénation. L'accès à l'adultité ne se déploie pas dans la perspective biopsychologique d'un accès à la maturité mais dans l'horizon axiologique d'un devenirmajeur, au sens kantien du terme. L'autorité est donc nécessaire. Il faudrait montrer, si nous en avions le temps, que la pire des injustices en éducation est l'abandon. J'ai montré dans mes travaux sur la sanction comment les rhétoriques qui plaident pour une éducation sans sanction oublient non seulement les leçons de l'histoire en réitérant les utopies déçues du libertarisme mais aussi et surtout comment elles abandonnent à d'autres le soin de sanctionner (sanction sociale, sanction pénale, sanction policière...) et qu'il y a dans cet abandon quelque chose de terrible, qui est l'oubli d'une promesse : celle d'avoir à répondre ?

Le respect, comme condition et comme horizon

L'autorité est nécessaire mais il ne suffit pas qu'elle soit nécessaire pour s'exercer; encore faut-il, nous l'avons vu, qu'elle soit perçue comme légitime. Toute la question est là aujourd'hui: l'autorité est posée comme objectivement nécessaire et subjectivement vécue, de plus en plus souvent, comme illégitime.

Il faut comprendre le rejet actuel, dans nos sociétés, de l'autorité et plus largement de toutes les formes relationnelles dissymétriques. Il ne faut pas se tromper de diagnostic. C'est la lente pénétration des valeurs démocratiques et la "passion de l'égalité » (Tocqueville) qui en découle qui menacent les rapports d'autorité au sein des espaces intermédiaires. C'est la lente pénétration des valeurs de liberté et d'égalité, valeurs liés à l'idéal démocratique, qui disqualifie aujourd'hui l'ensemble des rapports hiérarchiques au sein de l'institution scolaire et, par là, la possibilité même d'enseigner qui repose précisément sur un rapport asymétrique.

27 L'espace scolaire contemporain est travaillé par une tension que l'on peut résumer de la façon suivante: l'adolescent est de plus en plus souvent pensé dans une sorte d'éloignement anthropologique puisqu'il est tout sauf un adulte miniature (il y a une culture jeune, une mode jeune, un langage jeune...) et, dans le même moment, il est défini dans une très forte proximité politique à l'adulte (comme en témoignent la 
déclaration des droits de l'enfant ou encore les droits des lycéens dans la législation scolaire actuelle...). L'enfant est appréhendé à la fois comme semblable et différent. Il nous faut alors penser, de manière corrélative, une posture éducative qui assume et soutienne cette dialectique de l'identité et de l'altérité.

Il ne faut pas faire une lecture nostalgique de la crise de l'éducation, y voir le début d'un déclin irrémédiable et en appeler à un retour au statu quo ante. La crise de l'éducation est liée au choix de la démocratie. C'est une "crise structurelle ", comme l'explique très justement Alain Renaut, car elle découle de notre choix même des valeurs de liberté et d'égalité ${ }^{8}$.

Si l'autorité respecte, si l'autorité génère du respect, elle appelle aussi, dans les sociétés gagnées par les valeurs d'égalité et de liberté, comme condition de son déploiement l'existence d'une communauté respectueuse entre le maître et les élèves. Dans cette perspective, le respect doit être entendu dans une acception minimale. Aussi, la voie ouverte par Patrick Pharo, qui place son travail sous le signe d'une "sociologie sémantique ", nous semble tout à fait pertinente. "Le respect, écrit-il, est une position pratique de la première personne qui consiste pour celle-ci à limiter sa liberté d'action de façon à ne pas porter atteinte à la valeur éminente qu'elle reconnaît à une personne ou à un objet, ou qu'une personne accorde à un objet $"{ }^{9}$.

Cette définition présente un triple intérêt.

31 1. C'est une définition nécessaire qui se contente de préciser les composantes sans lesquelles le concept de respect ne serait plus le concept de respect. Ce minimalisme nous intéresse.

2. Elle ne pose pas comme nécessaire que le sujet respectueux s'efface entièrement devant ce qu'il respecte, et donc laisse ouvert la possibilité d'une attitude respectueuse entre égaux.

33 3. Enfin, en faisant du respect une position spécifique dans les positions pratiques de première personne, elle permet de comprendre la dynamique relationnelle et comment, par exemple, le respect peut devenir estime, bienveillance, considération...

Elle nous permet de comprendre comment de la réciprocité respectueuse entre personnes peut naître une relation d'autorité, ès qualités, entre un élève et un maître. Relation qui s'abolira un jour pour permettre une nouvelle rencontre, rencontre entre deux personnes libres et respectueuses inscrites, désormais, dans un stricte rapport d'égalité. Telle est la dialectique silencieuse qui est au cœur de la relation éducative. Tel est le chemin de l'éducation, il part du respect pour revenir au respect. Plus précisément, il part du respect comme auto-limitation de son agir pour aller jusqu'au respect, entendu alors « comme capacité à traiter autrui comme soi-même et soi-même comme autrui », selon la formule ricœurienne d'olivier Abel ${ }^{10}$.

\section{NOTES}

1.. H. ARENDT, La Crise de la culture, Paris, Gallimard/Folio, 1995, p. 123. 
2.. H.-G. GADAMER, Vérité et méthode. Les grandes lignes d'une herméneutique philosophique, Paris, Éditions du Seuil, 1996, p. 300-301.

3.. P. RICœUR, « Les paradoxes de l'autorité », in Quelle place pour la morale?, Paris, Desclée de Brouwer, 1996, p. 81.

4.. J. LOCKE, Quelques pensées sur l'éducation, Paris, Librairie Vrin, 1992, paragraphe 94.

5.. M. GAUCHET, « Démocratie, éducation, philosophie », in Pour une philosophie politique de l'éducation, Paris, Bayard, 2002, p. 37.

6.. Nous nous inspirons ici de la pensée d'Olivier REBOUL in Les Valeurs de l'éducation, Paris, PUF, 1992, p. 167-171. Olivier Reboul définit l'infantile en sept points, nous en proposons cinq qui définissent un ensemble de rapports (au monde, au temps, à autrui, à soi et au pouvoir).

7.. E. PRAIRAT, Sanction et socialisation, Paris, PUF, $2^{\mathrm{e}}$ édition, 2002.

8.. A. RENAUT, La Libération des enfants. Contribution philosophique à une histoire de l'enfance, Paris, Bayard/Calmann-Lévy, 2002, p. 24-37.

9.. P. PHARO, La Logique du respect, Paris, Les Éditions du Cerf, 2001, p. 86.

10.. O. ABEL, Paul Ricœur, la promesse et la règle, Paris, Éditions Michalon, Coll. « Le bien commun », p. 69.

\section{RÉSUMÉS}

Il y a une dialectique du respect et de l'autorité au cœur du travail éducatif, c'est cette dialectique silencieuse qui permet l'avènement d'un alter ego. Le chemin de l'éducation part du respect pour aller jusqu'au respect; plus précisément, il part du respect entendu comme simple autolimitation de son agir pour aller jusqu'au Respect entendu, cette fois, comme souci de l'autre, « comme capacité à traiter autrui comme soi-même et soi-même comme autrui », selon la formule ricœurienne d'Olivier Abel.

A dialectic of respect and authority exists in teaching and this silent dialectic allows the emergence of an alter ego. At school, the child goes from respect to respect or more precisely, one goes from respect understood as a simple self-limitation of behaviour to respect understood as care for the other, "as a capacity of treating the other as oneself and oneself as the other", according to a formula close to Ricœur's thought.

\section{AUTEUR}

\section{EIRICK PRAIRAT}

Eirick Prairat est Professeur de sciences de l'éducation à l'Université de Nancy 2 et responsable de la collection « Questions d'éducation et de formation » aux Presses Universitaires de Nancy. Il a récemment publié aux Presses Universitaires de France Sanction et socialisation (2002). Il publie à la rentrée (octobre 2003), chez le même éditeur, dans la collection « Que-sais-je ? ", La Sanction en éducation. 